\title{
Tabularia
}

TABULARIA Sources écrites des mondes normands médiévaux Guillaume de Volpiano : Fécamp et l'histoire normande | 2003

\section{Une étape décisive dans l'éveil des activités historiographiques au service du siège de Rouen : l'épiscopat de Maurille, moine de Fécamp}

A decisive step in the awakening of historiographic activities in the service of the Episcopal see of Rouen: the Episcopate of Maurille, a monk in Fécamp

\section{Louis Violette}

\section{OpenEdition Journals}

\section{Édition électronique}

URL : http://journals.openedition.org/tabularia/1878

DOI : 10.4000/tabularia.1878

ISSN : 1630-7364

Éditeur :

CRAHAM - Centre Michel de Boüard, Presses universitaires de Caen

\section{Référence électronique}

Louis Violette, «Une étape décisive dans l'éveil des activités historiographiques au service du siège de Rouen : l'épiscopat de Maurille, moine de Fécamp », Tabularia [En ligne], Guillaume de Volpiano : Fécamp et l'histoire normande, mis en ligne le 23 mai 2003, consulté le 30 avril 2019. URL : http:// journals.openedition.org/tabularia/1878; DOI : 10.4000/tabularia.1878 


\title{
Une étape décisive dans l'éveil des activités historiographiques au service du siège de Rouen: l'épiscopat de Maurille, moine de Fécamp
}

\section{A decisive step in the awakening of historiographic activities in the service of the Episcopal see of Rouen: the Episcopate of Maurille, a monk in Fécamp}

\author{
Louis VIOLETTE \\ Lycée Jeanne d'Arc, Rouen \\ louis_violette@hotmail.com
}

Résumé :

Le siècle de Guillaume de Volpiano est celui où s'éveillent à Rouen les activités historiographiques au service du siège épiscopal. Celles-ci se développent à partir de l'arrivée sur le siège de Rouen de Maurille, auparavant moine de Fécamp. Nous examinerons d'abord la situation de l'Église de Rouen au XI ${ }^{e}$ siècle, pour souligner ensuite les liens étroits qui existent entre l'entreprise de réforme commencée par Guillaume de Volpiano à Fécamp et qui s'est étendue à l'ensemble du duché dans la seconde moitié du XIe siècle, et la rédaction de textes consacrés à l'histoire des archevêques à la cathédrale de Rouen, qui a commencé sous l'épiscopat de Maurille.

Mots-clés: Acta archiepiscoporum Rothomagensium, Annales de Rouen, Chronique métrique des archevêques de Rouen, historiographie, Maurille, Réforme grégorienne.

\begin{abstract}
:
The century of William of Volpiano coincides with the emergence in Rouen of historiographic activities in the service of the Episcopal see, which developed after the arrival at the Episcopal see of Maurille, who used to be a monk in Fécamp. We shall first examine the situation of the Church of Rouen in the 11th century, then dwell on the close links between the reform initiated by William of Volpiano in Fécamp and which then spread to the whole Duchy in the second half of the 11th century, and finally the writing of texts dedicated to the history of archbishops in the Cathedral of Rouen, which began under the Episcopate of Maurille.
\end{abstract}

Keywords: "Acta archiepiscoporum Rothomagensium ", "Annals of Rouen", "Metrical Chronicle of archbishops of Rouen», historiography, Maurille, Gregorian Reform.

Le siècle de Guillaume de Volpiano est celui où s'éveillent les activités historiographiques au service du siège de Rouen. Celles-ci, qui ne se développent qu'à partir de la seconde moitié du XI ${ }^{\mathrm{e}}$ siècle (nous ne possédons aucun texte antérieur à cette époque sur les archevêques de Rouen), c'est-à-dire un bon demi-siècle après l'arrivée de Guillaume de Volpiano à Fécamp, sont pourtant assez directement liées au courant de réforme qu'il a impulsé en Normandie, puisque c'est avec l'arrivée de 
Maurille qu'elles débutent. Nous verrons dans un premier temps quelle est la situation de l'Église de Rouen au début du XIe siècle, puis dans quelles conditions se sont développées les activités historiographiques au service du siège de Rouen, pour souligner enfin l'importance des liens entre l'entreprise de réforme commencée par Guillaume à Fécamp puis développée par les grégoriens et ces activités historiographiques.

\section{Situation de l'Église de Rouen au début du XI ${ }^{\mathrm{e}}$ siècle}

Lorsque Guillaume de Volpiano prend en mains les destinées de Fécamp, en 1001, comment caractériser l'état de l'Église de Rouen? Cette Église, qui est une des plus anciennes de la France du nord (son existence est attestée dès 314, année où l'évêque Avitianus assiste au concile d'Arles en compagnie de son diacre Nicetius), est aussi celle des Églises de Normandie qui a le moins souffert des invasions scandinaves: alors qu'il y a eu interruption de la présence des évêques sur les sièges épiscopaux de la Normandie occidentale, il n'y a pratiquement pas eu à Rouen de vacance du siège épiscopal. Et l'archevêque de Rouen est à nouveau, à la fin du Xe siècle, à la tête d'une province ecclésiastique dont les structures ont été peu à peu reconstituées comme en témoigne la charte de Richard Ir donnée pour Fécamp vers $990^{1}$. Cette charte concédée par le duc est octroyée en présence de sept évêques: l'archevêque de Rouen, Robert, et ses six suffragants: Raoul de Bayeux, Hugues de Coutances, Gérald d'Evreux, Roger de Lisieux, Atso de Sées, et Norgod d'Avranches. Comme l'avait souligné Lucien Musset $^{2}$, ce document est le premier qui atteste à nouveau la présence d'un titulaire sur chacun des 6 sièges suffragants que compte la province de Rouen. Le christianisme a donc repris pied partout en Normandie, puisque l'Église ne dote d'évêques que les cités possédant une communauté chrétienne importante. L'archevêque se trouve donc à la tête d'une province ecclésiastique à nouveau en état de marche. Mais que dire à la même époque de la situation de l'Église métropolitaine?

Cette Église est dans un état difficile. Son patrimoine apparaît très réduit au $\mathrm{X}^{\mathrm{e}}$ siècle et encore au début du XI $\mathrm{XI}^{\mathrm{e}}$ siècle : ce n'est qu'entre 1028 et 1033 que, grâce à une grande charte octroyée par le duc Robert le Magnifique (1027-1035) à son oncle, l'archevêque Robert (990-1037) $)^{3}$, le patrimoine atteint à nouveau les proportions qu'il avait à l'époque carolingienne. Cependant, après cette charte, et grâce à ce patrimoine retrouvé, le même archevêque Robert a pu mettre en chantier une nouvelle cathédrale, la cathédrale romane, dont la construction s'étale de l'époque de la charte

1. Fauroux, Marie, Recueil des Actes des ducs de Normandie, Mémoires de la Société des Antiquaires de Normandie, t. XXXVI, Caen, 1961, n 4, p. 72-74 [ci-après, FAUrouX, Recueil...].

2. MUSSET, Lucien, «Un millénaire oublié: la remise en place de la hiérarchie épiscopale en Normandie autour de 990 », in Mélanges Marcel Pacaut, Pierre GUICHARD, Marie-Thérèse LORCIN, Jean-Michel Poisson et M. Rubellin (éd.), Lyon, Presses universitaires de Lyon, 1994, p. 563-573.

3. Nous possédons deux versions de ce texte dans le cartulaire de la cathédrale de Rouen (Bibl. mun. Rouen, ms Y 44, Omont 1193) qui ont été éditées par Marie FAUROUX, Recueil..., n 66 et 67. 
de restitution à 1063. Son œuvre en ce domaine est poursuivie par Maurille, qui devient en 1054/1055 archevêque de Rouen, en remplacement du neveu de Robert, Mauger, qui a été déposé par un concile provincial ${ }^{4}$.

Par Maurille, archevêque de 1054/1055 à 1067, le vent de réforme qui a soufflé dans un certain nombre de monastères normands dans la première moitié du XI ${ }^{\mathrm{e}}$ siècle, s'impose enfin à la tête de l'Église de Rouen. Si l'œuvre des réformateurs grégoriens en faveur d'une plus grande rigueur des mœurs du clergé nous est familière, leur œuvre en faveur des activités historiographiques est moins connue. C'est pourtant lorsque s'impose à Rouen la réforme grégorienne que s'y développent pour la première fois des activités historiographiques au service de l'Église de Rouen.

\section{L'éveil des activités historiographiques au service de l'Église de Rouen au XI' ${ }^{\mathrm{e}}$ siècle}

\section{Trois textes d'histoire rédigés par les chanoines de Rouen}

Les premiers travaux historiographiques dont nous disposons sur l'Église de Rouen sont deux textes écrits par les chanoines de la cathédrale dans la seconde moitié du XI ${ }^{\mathrm{e}}$ siècle: une histoire des archevêques de Rouen, les Actes des Archevêques de Rouen $^{5}$ et les Annales de Rouen ${ }^{6}$. Ces deux textes appartiennent à des genres différents:

- les Actes des Archevêques de Rouen appartiennent à un genre qui a fleuri surtout à l'époque carolingienne, les gesta episcoporum ${ }^{7}$ ou gestes d'évêques, textes qui se composent d'une série de notices sur les évêques d'un siège, en ordre chronologique, en commençant à la fondation du siège et en allant jusqu'au temps de la narration. C'est le second texte de ce genre en Normandie, puisqu'il y a eu à l'époque carolingienne un texte de gesta rédigé dans cette province: les Gesta abbatum Fontanellen$\operatorname{sium}^{8}$, mais on ne relèvera guère de trace d'une influence d'un texte sur l'autre.

4. Olivier GUILLOT a discuté les raisons et les modalités du renversement de Mauger ainsi que la datation traditionnelle de celui-ci dans "La libération de l'Église par le duc avant la conquête", in Histoire religieuse de la Normandie, Chambray, CLD, 1981, p. 71-86.

5. Ce texte nous est parvenu dans deux versions manuscrites, l'une dans le Livre d'Ivoire de la Cathédrale de Rouen (Bibl. mun. Rouen, ms Y 27, Omont 1405, p 26-36) et l'autre dans le Livre Noir de SaintOuen (Bibl. mun. Rouen, ms Y 41, Omont 1406, fol 1-12). Ce texte a été édité deux fois d'après la version, plus longue, du Livre Noir par dom Edmond MARTENE, Veterum scriptorum collectio nova, 1707, pars II, p. 233-248, et par dom Jean MABILLON, Vetera analecta, 1723, p. 222-226. Nous en avons donné une traduction française dans La Cathédrale de Rouen, seize siècles d'Histoire, Jean-Pierre CHALINE (dir.), Rouen, Société d'Histoire de Normandie, 1996, p. 49-60.

6. Nous ne possédons plus de manuscrit d'époque des Annales de Rouen, dont le texte a été édité par LABBÉ, Philippe, Novae bibliothecae manuscriptorum librorum, Paris, Sebastien Cramoisy, 1657, t. 1, p. 364-390.

7. Sur le genre des gesta, voir Michel Sot, Gesta episcoporum, gesta abbatum, Turnhout, Brepols, (coll. Typologie des sources du Moyen Âge occidental, fasc. 37), 1981, 57 p.

8. Texte édité par dom Fernand LOHIER et dom Jean LAPORTE, Gesta sanctum patrum Fontanellensis coenobii, Rouen-Paris, Société de l'Histoire de Normandie, A. Lestringant et A. Picard, 1936, XLI143 p., récemment traduit sous le titre Chronique des abbés de Fontenelle (Saint-Wandrille), éd. Pascal PrADIÉ, Paris, Les Belles-Lettres («Les classiques de l’Histoire de France au Moyen Âge»), 1999, 283 p. 
- les Annales de Rouen appartiennent à un autre genre, encore plus ancien, qui consiste à relever année après année des événements jugés importants et à les intégrer dans une chronologie. Ces textes d'Annales comportent de nombreux éléments de comput, et se présentent au départ comme des tables de concordance entre des événements considérés comme ayant valeur de repère, les règnes des rois, des empereurs, des papes, des ducs et pour un certain nombre d'années s'ajoutent de très brèves notations d'événements jugés comme importants.

À ces travaux appartenant à des genres historiques bien identifiés, il faut joindre un autre travail appartenant à un genre qu'on peut également qualifier d'historique, quoique son contenu factuel soit très maigre, et bien qu'il s'agisse d'un poème: une Chronique métrique des archevêques, c'est-à-dire un poème qui consacre deux vers à chacun des archevêques ayant occupé le siège depuis sa fondation et que l'on trouve à la suite du texte des Acta dans le Livre d'Ivoire de la cathédrale et dans le Livre Noir de Saint-Ouen ${ }^{9}$.

\section{Des diffusions différentes}

Ces textes auront en Normandie des diffusions diverses: les Actes des Archevêques de Rouen qui forment un texte beaucoup plus développé, susceptible d'intéresser plus facilement un lecteur moderne qu'un texte d'annales, dont les notations sont très brèves, ont en fait eu à l'époque un rayonnement beaucoup plus faible. En effet, le texte des Acta n'a été recopié qu'à Saint-Ouen, abbaye voisine et rivale de la cathédrale en prestige, alors que le texte des Annales de Rouen se retrouve dans tous les établissements de la province ecclésiastique. Pourquoi ce contraste? Les textes d'annales sont beaucoup plus souples d'utilisation: dans chaque établissement où il est recopié, un tel texte est enrichi de notations nouvelles qui donnent aux annales de l'établissement cette touche d'originalité qui permet de l'attribuer à tel ou tel monastère. Chacun peut en fait réutiliser un texte d'annales pour son propre usage. Ainsi dérivent des Annales de Rouen toute une série d'annales normandes des $\mathrm{XI}^{\mathrm{e}}$, XII ${ }^{\mathrm{e}}$ et XIII ${ }^{\mathrm{e}}$ siècles : Annales de Saint-Évroult ${ }^{10}$, Annales de Robert de Torigni ${ }^{11}$, Annales de Jumièges ${ }^{12}$... Un texte d'annales est donc par nature fait pour être réutilisé : il fournit des cadres chronologiques, des listes de synchronismes entre les dates de règnes des rois, des empereurs et des papes que l'on peut réemployer dans chaque établissement pour se doter d'annales locales.

9. Bibl. mun. Rouen, ms 1405, p 36-40 et Bibl. mun. Rouen, ms 1406, fol 12-16. Le texte de la Chronique métrique des archevêques a été éditée à la suite des Acta archiepiscoporum rothomagensium par dom Edmond MARTENE, à partir de la version du Livre Noir (MARTENE, 1707).

10. Annales de Saint-Evroult, éd. Auguste LePrÉvost, in Historiae ecclesiasticae libri tredecim, Paris, Société de l'Histoire de France, J. Renouard, 1838-1855.

11. Chronique de Robert de Torigni, éd. Léopold Delisle, Rouen, Société de l'Histoire de Normandie, t. 1, 1872, p. 2-90 et 119-369.

12. Annales de Saint-Pierre de Jumièges, chronique universelle des origines au XIII ${ }^{\mathrm{e}}$ siècle, édition et traduction par dom Jean LAPORTE, Rouen, Lecerf, 1954, 128 p. 


\section{La diffusion des Acta}

Un texte de gesta episcoporum au contraire est moins facilement réutilisable: il ne concerne en fait que l'établissement qui l'a produit. Ce qui est étonnant, ce n'est pas que les Acta n'aient pas été davantage diffusés, mais bien qu'ils aient été recopiés à l'abbaye de Saint-Ouen, puisque nous trouvons deux versions du texte, l'une dans le Livre d'Ivoire de la cathédrale, l'autre dans le Livre Noir de Saint-Ouen. Pourquoi les moines de Saint-Ouen ont-ils donc recopié les Actes des archevêques en tête d'un lectionnaire - qui est par ailleurs un ouvrage d'une grande unité - et qui ne contient hors cela que des textes hagiographiques?

Si les moines ont pu s'intéresser à cette histoire des évêques, c'est d'abord parce que l'abbaye de Saint-Ouen et la cathédrale étaient deux établissements dont l'histoire était largement liée : jusqu'à la fin de l'époque carolingienne, comme en témoigne une charte d'invention de reliques donnée par Riculfe en 872 et dont l'original nous est parvenu ${ }^{13}$, l'évêque de Rouen est en même temps l'abbé de Saint-Ouen. Pourtant, depuis le début de la période normande, les deux établissements sont séparés: les moines semblent alors avoir leur propre maitre, un abbé. L'histoire des archevêques leur était-elle à la fin du XI siècle encore nécessaire pour écrire leur propre histoire? Nous ne le croyons pas : il n'y a pas trace d'aménagement du texte dans ce sens. La raison de cette duplication du texte réside dans les ajouts que les moines ont pu y faire. Les moines de Saint-Ouen en recopiant le texte des Actes des archevêques l'ont augmenté à ses deux extrémités: ils inscrivent au début du texte comme premier évêque de Rouen le martyr Nicaise dont ils ont récupéré le corps en 1032, et ils relatent à la fin du texte la violente altercation qu'ils ont eue avec l'archevêque Jean d'Avranches, en reportant tous les torts de l'affrontement sur l'archevêque, après quoi ils ajoutent une notice sur son successeur, Guillaume Bonne-Âme. Si le récit de l'altercation entre l'archevêque et les moines est très développé et haut en couleurs, il ne me parait cependant pas central : l'essentiel du projet est de faire reconnaître Nicaise pour le premier évêque de Rouen. Nous en avons deux signes:

- les moines ont recopié à la suite du texte des Acta la Chronique métrique des archevêques, comme dans le Livre d'Ivoire de la cathédrale, et ont augmenté cette chronique métrique de 32 vers sur Nicaise,

- les moines ont placé ces deux textes en tête d'un dossier sur Nicaise: on trouve à leur suite deux versions de la Passio Nicasii, l'une en prose (B.H.L. 6082) et l'autre en vers (B.H.L. 6083), et un récit de la translation des reliques de Nicaise à Rouen en 1032 (B.H.L. 6084). Ce qui les intéresse donc dans ces textes d'histoire des évêques, c'est surtout d'y intégrer le nom de Nicaise, martyr du Vexin, dont ils ont recueilli les reliques. Les moines de Saint-Ouen, pour valoriser les reliques de Nicaise, et pour susciter la piété des fidèles rouennais à son égard, le présentent en effet comme un évêque envoyé du pape saint Clément auprès des Rouennais ${ }^{14}$. Sur ce point, ils ont

13. Rouen, Arch. dép. Seine-Maritime, 14 H 156.

14. Sur l'argumentation développée par les moines en direction des habitants de Rouen pour leur faire adopter le culte de saint Nicaise comme celui d'un saint local, voir VIOLETTE, Louis, « Nicaise, du 
su se montrer convaincants, puisqu'au XII siècle le nom de Nicaise sera ajouté en tête de la liste des évêques de Rouen dans le Livre d'Ivoire. Pour charpenter leur argumentation, ils ont construit un dossier solide, composé de 5 pièces différentes qui se suivent au début du Livre Noir de Saint-Ouen : le texte des Acta archiepiscoporum Rothomagensium augmenté d'un passage sur Nicaise, la Chronique métrique des archevêques augmentée en son début de 32 vers sur Nicaise, et trois textes hagiographiques: une passion en prose, une passion en vers et le texte de la translation à Rouen des reliques de Nicaise. La raison de la reprise par les moines de Saint-Ouen du texte des Acta et de la Chronique métrique apparaît alors clairement : elle vise à faire connaître Nicaise comme un proto-évêque de Rouen, et les moines recopient les deux ouvrages écrits par les chanoines de la cathédrale sur l'histoire de leurs évêques pour les intégrer dans un dossier hagiographique, dont ils contribuent à renforcer la cohésion.

Le texte de la Chronique métrique a, lui, connu une diffusion un peu plus large que celle des Acta, mais nettement moins importante que celle des Annales. La chronique a en effet été recopiée par les moines de Saint-Ouen comme les Acta, mais elle l'a été aussi dans d'autres établissements normands : elle a d'abord été intégrée par Orderic Vital dans le chapitre de son Histoire Écclésiastique qu'il a consacrée aux archevêques (livre 5 , chap. 9) ${ }^{15}$ ce qui assure à ce poème la diffusion qui a été celle de l'œuvre d'Orderic, et elle a également été recopiée et continuée à Mortemer au XIII siècle (BnF, ms lat. 4863, fol. 112). Si cette diffusion est donc plus modeste que celle des Annales, il faut tout de même remarquer que, dans chacun des établissements cités, ce poème a été continué: c'est une œuvre qui a eu, dans les trois établissements concernés (la cathédrale, Saint-Ouen, Mortemer) une trentaine de continuateurs, indice d'un intérêt qui s'est maintenu pendant environ deux siècles (c'est au XIII siècle que s'arrêtent les continuations).

\section{Les raisons de l'éveil d'une historiographie pontificale à Rouen}

Mais si nous nous interrogeons sur les raisons qui poussent à recopier tel ou tel texte, il faudrait aussi se poser la question de savoir pourquoi ont été rédigés ces textes par leurs auteurs initiaux.

\section{La datation de ces textes}

Ces auteurs sont inconnus, mais nous pouvons dater assez précisément ces trois textes : la première écriture des Acta du Livre d'Ivoire se termine par une notice sur

martyr du Vexin au saint rouennais, valorisation de reliques par l'hagiographie» in Autour des morts, Mémoire et identité, Actes du V e colloque international sur la Sociabilité, 19-21 novembre 1998, Publications de l'Université de Rouen n²296, 2001, p. 377-386.

15. The Ecclesiastical History of Orderic Vitalis, éd. Marjorie CHIBNALL, Oxford (Oxford University Press), 1969, vol. 3, p. 48-96 [ci-après, ORDERIC VITAL]. 
Jean d'Avranches qui ne mentionne pas sa mort. Jean ayant été archevêque de 1067 à 1079, la fourchette se situe dans la période 1067-1079 pour les Acta. La Chronique métrique des archevêques, dont la première main s'arrête dans le Livre d'Ivoire de la cathédrale sur la notice de Maurille, et qui a été copiée à la suite du texte des Acta peut être datée de la même époque que ceux-ci.

Pour les Annales de Rouen, nous sommes un peu plus gênés, car nous ne possédons plus de manuscrit d'époque de ce texte. Ce qui servira alors de point de repère, c'est la dernière notice commune à ces Annales et aux suivantes: il s'agit de celle de la mort de Guillaume le Conquérant en 1087. Les Annales seraient donc un texte postérieur aux Acta d'une dizaine d'années. Mais il y a probablement eu plusieurs étapes dans la rédaction ainsi que le suggère l'examen d'un texte des Annales de Fontenelle dans un manuscrit de La Haye (le ms $124 \mathrm{E} 28$ fol 1 à 11) ${ }^{16}$. Des Annales de Fontenelle, qu'il ne faut pas confondre avec celles qui ont été rédigées à l'époque carolingienne, sont dérivées des Annales de Rouen, mais elles n'offrent qu'une chronologie incomplète des évêques de Rouen et elles ne suivent les Annales de Rouen que jusqu'à l'année 1066, après quoi elles divergent. On peut donc penser que les Annales de Rouen ont été élaborées avant 1066, date à laquelle on a commencé à les recopier, ce qui n'a pas empêché de continuer à les travailler à Rouen, comme en témoigne la version plus développée qui circule à partir de 1087.

\section{Des textes rédigés au temps du triomphe des grégoriens}

Les travaux historiographiques sur les évêques ont donc débuté dans la seconde moitié du XI ${ }^{\mathrm{e}}$ siècle, avant 1066 pour les Annales, entre 1067 et 1079 pour les Actes des archevêques et la Chronique métrique des archevêques, soit sous les épiscopats de Maurille (1055-1067) et de Jean d'Avranches (1067-1079). Cette période d'intense effort historiographique coïncide donc avec le triomphe du parti grégorien à Rouen. Maurille, qui devient archevêque en 1054/1055 à la suite de la déposition de l'archevêque Mauger jugé indigne, est le premier réformateur grégorien à occuper le siège de Rouen. Cet homme a eu un parcours qui ne saurait nous laisser indifférent: originaire de Lorraine, il a d'abord été moine à Fécamp avant de monter sur le siège épiscopal. C'est donc par Fécamp que la réforme, qui s'est développée au XIe siècle dans le monde monastique, gagne vers le milieu du siècle le clergé séculier, et c'est sous l'autorité d'un homme qui a été moine à Fécamp que sont rédigées les premières Annales de Rouen. Et c'est sous son successeur, autre réformateur énergique, qu'ont été rédigés les Actes des archevêques.

16. Ce manuscrit a été présenté par GUMBERT, Joan-Peter, «Un manuscrit des Annales de Saint-Wandrille retrouvé», Bibliothèque de l'École des Chartes, 136, 1978, p. 74-75. Nous en avons donné une transcription dans L'Église métropolitaine de Rouen pendant la première période normande, Thèse de doctorat, Paris X-Nanterre, 1994, p. 455-461. 


\section{Des textes militants}

C'est donc aux grégoriens que nous devons les premières tentatives pour écrire l'histoire des évêques de Rouen: ces réformateurs sont des hommes exigeants en tous domaines, aussi bien dans celui de la discipline des clercs, auxquels ils entendent imposer le célibat, que dans celui de la culture, et nous ne nous en plaindrons pas. La notice la plus développée du texte des Acta, et de loin, est celle qui est consacrée à Maurille qui est présenté comme un modèle de vertu en tous domaines. C'est d'ailleurs la seule notice du texte qui constitue une véritable fiche biographique, présentant les origines et les étapes de la carrière du prélat, élève à Reims, puis à Liège, écolâtre à Halberstadt, puis moine à Fécamp, d'où il part pour tenter une expérience de vie érémitique en Italie, où il est sollicité pour prendre la direction d'un monastère florentin, direction à laquelle il renonce devant les résistances que rencontrent ses projets de réforme. Après quoi, il rentre à Fécamp, d'où le tirera le duc pour le placer sur le siège de Rouen. Ce texte juge l'œuvre des archevêques qui se sont succédé sur le siège avec des critères grégoriens, ainsi insiste-t-il particulièrement sur l'œuvre de Maurille en faveur de la réforme, présentée comme un retour aux sources: «il célébra un concile dans l'église de Rouen pour que la chasteté soit observée et que les autres institutions des saints Pères, reléguées négligemment au second plan par l'incurie des pasteurs, soient fermement rétablies", et plus loin: «il fit de nombreux bienfaits pour le rétablissement de la loi chrétienne et de pratiques religieuses de l'Église.»

Mais cet engagement en faveur de la réforme a une contrepartie. Le texte dresse pour les prélats de la période normande des portraits contrastés: certains, sont encensés (le cas le plus voyant est celui de Maurille) alors que d'autres sont condamnés, ainsi les archevêques Hugues (942-989) ou Mauger (1037-1055). Et ces jugements négatifs portés sur certains prélats se retrouvent dans les notations pourtant brèves des Annales: Hugues est dit «monachus habitu, non opere» («moine par l'habit et non par les œuvres»), et à l'année 1036: Obiit Rotbertus arch. Succedit (sic) Malginus qui culpa sua exigente depositus est. Au contraire, pour les prélats des périodes anciennes, dont on sait en fait peu de choses, les louanges sont assez généralement distribuées, comme il est habituel dans ce type d'ouvrage, qui a pour finalité de couvrir de gloire la lignée des évêques, gloire qui forme comme un capital que l'évêque titulaire du siège peut utiliser pour affirmer son autorité.

\section{Des jugements que l'on peut nuancer}

En fait, à un certain nombre de signes, on peut deviner que ces jugements doivent être nuancés pour se faire une image plus exacte de l'histoire du siège de Rouen pendant la période normande: si Maurille a joué un rôle important en relançant l'activité conciliaire ou en achevant la cathédrale nouvelle qu'il dédicace en 1063, l'œuvre accomplie par ses prédécesseurs ne doit pas être méconnue : l'archevêque Robert, que les Acta traitent avec indulgence mais rapidement, a eu un rôle considérable dans le renforcement de la puissance de l'Église de Rouen : c'est lui qui obtient du duc 
Robert vers 1028-1033 une grande charte qui permet à l'Église de Rouen de retrouver un patrimoine comparable à celui qu'elle avait à l'époque carolingienne et c'est lui qui commence la reconstruction de la cathédrale. Et des cas comme ceux des archevêques Hugues (942-989) et Mauger (1037-1055) vaudraient d'être réexaminés: en fait, si Robert a eu un rôle capital pour la renaissance temporelle de l'Église de Rouen, le rôle joué par Hugues a sans doute été lui aussi important, malgré ce que laisse entendre l'histoire officielle rouennaise. Il est probable, comme l'a suggéré Félice Lifshitz, qu'il a joué un rôle politique important, en particulier au moment des troubles liés à la minorité de Richard I Ir. Dans ce contexte, il a, selon elle, pu jouer le rôle de chef d'un parti favorable à l'intégration de la principauté normande dans l'ensemble franc, du fait de ses liens avec l'abbaye de Saint-Denis dont il avait été tiré par Guillaume Longue-Épée. Mais il joue aussi à Rouen un rôle important pour la renaissance des activités intellectuelles et pour la reconstitution de la puissance spirituelle de cette Église : c'est à lui qu'il faut attribuer l'initiative de la promotion du culte de saint Romain, personnage pour lequel il commande une vita à un certain Gérard de Soissons que Felice Lifshitz propose d'identifier à Gérard de Brogne, ce qui sera à l'origine d'une promotion systématique de la réputation de saint Romain qui n'apparaissait pas encore dans le haut Moyen Âge comme un grand saint rouennais ${ }^{17}$.

Il faut donc nuancer l'image négative de l'archevêque Hugues et admettre que cet homme qui a été condamné de façon abrupte par les grégoriens pour avoir distrait quelques biens d'Église en faveur de sa famille (le texte des Acta mentionne l'aliénation du domaine de Tosny au profit d'un membre de sa famille) ${ }^{18}$, a par ailleurs joué un rôle dans le redressement de son Église.

Et on peut même se demander si l'indigne Mauger, déposé par le duc qui a fait alliance avec les grégoriens, n'a pas eu comme principal crime de continuer à vivre à l'ancienne mode, dans le faste, comme son oncle Robert, au lieu d'être sensible aux exigences nouvelles que formulaient les réformateurs au milieu du $\mathrm{XI}^{\mathrm{e}}$ siècle...

\section{Des textes rédigés dans un contexte de renforcement du pouvoir archiépiscopal}

Quoi qu'il en soit, la seconde moitié du XIe siècle a vu fleurir à Rouen une historiographie épiscopale, en particulier sous les épiscopats de Maurille et Jean d'Avranches. Ces textes rédigés à une époque de réforme témoignent à la fois du renforcement de l'autorité de l'archevêque et des difficultés à la faire admettre. Autorité sur une province ecclésiastique qui, avec la conquête normande, a progressivement trouvé

17. Lifshitz, Felice, The Power of discours. The Normann conquest of Pious Neustria. A regional Case Study in Religious Historiography, Florida International University, Miami, 1994 (dacyl.).

18. Le texte de la notice des Acta sur Hugues: filios enim quamplures procreavit, ecclesiam et res ecclesiae destruxit. Todiniacum enim qui in dominicatu archiepiscopi erat, cum omnibus appendiciis suis fratri suo Radulfo, potentissimo viro, filio Hugo de Calvacamp, dedit, et ita a dominicatu archiepiscopatus usque in praesens alienavit. 
une cohésion nouvelle: la province ecclésiastique s'identifie depuis la seconde moitié $\mathrm{du} \mathrm{X}^{\mathrm{e}}$ siècle avec la nouvelle Normandie, à quelques exceptions près (à l'est, le diocèse de Rouen englobe la totalité du Vexin, alors que la frontière du duché s'arrête plus à l'ouest, sur le cours de l'Epte). Et dans cette principauté normande bien tenue en main par ses ducs, le pouvoir de l'archevêque est d'autant mieux affermi que la nomination des évêques dépend désormais dans toute la province ecclésiastique du même homme : le duc. Cette cohésion nouvelle de la province de Rouen est affirmée par le texte des Acta qui commence par une mention de la Notitia provinciarum qui énumère les Églises suffragantes de celle de Rouen ${ }^{19}$. Cette cohésion nouvelle de la province ecclésiastique se lit également dans le renouveau de l'activité conciliaire : c'est avec Maurille l'époque des premiers conciles normands, comme celui que mentionne le texte des Acta et qui réunit à Rouen en 1063 l'ensemble des évêques de la province ${ }^{20}$.

Nos textes historiographiques, rédigés pour exalter l'histoire du siège de Rouen, participent donc de cette conjoncture de renforcement de l'autorité métropolitaine des évêques, mais ne nous y laissons pas prendre: cette entreprise rencontre des résistances, qui provoquent même des conflits ouverts dès le pontificat de Jean d'Avranches : il a été en conflit pour des problèmes de préséance avec les moines de Saint-Ouen qui ne l'ont pas attendu pour célébrer la fête de leur saint patron en 1073, ce qui a provoqué une bagarre généralisée entre les moines et les gens de l'archevêque dans l'abbatiale à la suite de laquelle il a fallu solliciter l'arbitrage du duc ${ }^{21}$. Et le programme de réforme que le même archevêque a tenté de faire accepter lors d'un synode réuni en 1076 a de même provoqué la révolte des clercs qui, d'après Orderic, l'auraient chassé de l'église en lui jetant des pierres $^{22} \ldots$

L'émergence d'une historiographie épiscopale à Rouen est donc en même temps liée au fait que les archevêques prétendent à un meilleur contrôle de leur province

19. L'extrait de la Notitia provinciarum cité par les Acta: Galliae provinciae sunt decem et octo, quarum una exsistit Lugdunensis, quae vocatur secunda, in qua est nobilis et ampla civitas, quae vocatur Rodomus vel Rothomagus, super fluvium Sequanae sita. Hujus civitatis ecclesia in honore beatae et gloriosae Dei genetricis semper virginis Mariae est consecrata. Haec vero est metropolis. Sex enim sub se civitates episcopales continet, scilicet primam civitatem Baiocatarum, secundum civitatem Abrincatarum, tertiam civitatem Evatinorum, quae dicitur Ebroicas, quartam civitatem Salanum, quae vocatur Sagium, quintam civitatem Lexoviorum, sextam civitatem Constantinorum.

20. Il faut tout de même signaler que l'activité conciliaire avait recommencé dans la province de Rouen dès le pontificat de Mauger: nous avons conservé les actes d'un concile réuni par lui, qui ont été recopiés au XVII siècle par Sirmond sur un manuscrit en provenance du Mont-Saint-Michel et qui ont ensuite été publiés par Dom Guillaume BEssin, Concilia Rotomagensis Ecclesiae, Rouen, Vaultier, 1717, p. 40-45.

21. Les Annales de Rouen à l'année 1073 attribuent la responsabilité de l'affrontement aux moines et signalent que trois d'entre eux ont été envoyés dans d'autres monastères, à Fécamp, à Fontenelle et à Jumièges, mais les Acta du «Livre Noir», interpolés par les moines, indiquent que le roi Guillaume, lassé par l'intransigeance de l'archevêque, a finalement autorisé les moines à regagner leur abbaye et a condamné en conseil l'archevêque Jean à une amende de 300 livres.

22. «... Multumque contra impudicos presbyteros pro auferendis pelicibus concubinas eis sub anathemate prohiberet lapidibus percussus aufugit... » in ORDERIC VITAL..., t. 2, p. 200. 
et qu'ils s'appuient pour cela sur des prétentions historiques (la deuxième Lyonnaise remonte à l'époque romaine), et en même temps que cette montée en puissance de l'autorité du chef de la province ecclésiastique, qui ne va pas sans rencontrer de résistances, doit s'accompagner, pour surmonter ces résistances, d'un effort de justification idéologique qui passe par l'historiographie. 